\title{
Formal approaches to multi-agent sysems
}

\author{
Rineke Verbrugge • Barbara Dunin-Kȩplicz
}

Published online: 7 November 2008

The Author(s) 2008. This article is published with open access at Springerlink.com

In recent years, multi-agent systems have come to form one of the key technologies for software development. The Formal Approaches to Multiagent Systems (FAMAS) workshop series brings together researchers from the fields of logic, theoretical computer science and multi-agent systems in order to discuss formal techniques for specifying and verifying multiagent systems, including many subtle and not easy to formalize aspects of agency. FAMAS addresses the issues of logics for multiagent systems, formal methods for verification, e.g. model checking, and formal approaches to cooperation, multi-agent planning, communication, coordination, negotiation, games, and reasoning under uncertainty in a distributed environment.

The first workshop in the FAMAS series, FAMAS'03, took place in Warsaw in April 2003 as a satellite event of the European Conference on Theory and Practice of Software (ETAPS'03). A selection of contributed and invited papers was published in Fundamenta Informaticae as volume 63, issue 2,3 of 2004.

The second FAMAS workshop, FAMAS'06, took place in August 2006 at the Riva del Garda, in conjunction with the European Conference on Artificial Intelligence (ECAI'06). The best contributions resulted in the current special issue of the Journal of Autonomous Agents and Multiagent Systems.

FAMAS'07 was one of the agent workshops gathered together under the umbrella of Multiagent Logics, Languages and Organizations-Federated Workshops (MALLOW'007), taking place in September 2007 in Durham. Finally, FAMAS'09 will take place in Turin as a part of MALLOW'09.

\footnotetext{
R. Verbrugge ( $\varangle)$

University of Groningen, Groningen, The Netherlands

e-mail: rineke@ai.rug.nl

B. Dunin-Kęplicz

Institute of Informatics, Warsaw University, Warsaw, Poland

B. Dunin-Kęplicz

Institute of Computer Science, Polish Academy of Sciences, Warsaw, Poland

e-mail: keplicz@mimuw.edu.pl
} 
All research reported in the current volume is squarely related to practice, even if the formal approach is taken. Thus, authors of FAMAS contributions devote their attention to pressing practical problems such as supporting organizations in planning, security and effective communication. Since the first FAMAS edition, emphasis has been shifting to correspond to the situatedness of multi-agent systems in a dynamic environment. Also, quite a few authors take on the challenge to combine different logics or to investigate the relations between different logical viewpoints (e.g. temporal versus dynamic logic) in a methodologically sound manner.

\section{Verification and dynamical aspects of multi-agent systems}

In Hindriks and Meyer's "Toward a programming theory for rational agents", the focus is on an agent programming theory consisting of both an agent programming language and a corresponding logic to verify agent programs. The logic, "dynamic knowledge logic (DKL)" is inspired by dynamic epistemic logic, incorporating declarative goals. For the dynamic agent programming language, the authors provide both an operational and a denotational semantics. The two types of semantics are proved to be equivalent. Finally, the paper gives an interesting first step into formalizing the process of goal adoption, based on second-order goals.

Roos and Witteveen, in their paper "Models and methods for plan diagnosis", present a model-based diagnosis approach to plan diagnosis. They restrict themselves to the case in which failure of actions causes the failure of the plan as a whole, arguing that if a plan is correctly specified, then errors in its execution become manifest in the incorrect performance of at least one action. They present a formal framework for diagnosis, and show that their "mini-maxi diagnoses" can be computed efficiently.

\section{Communication and security in a multi-agent setting}

Orgun, Governatori and Liu provide a fruitful bridge from theory to practice in their contribution "Modal tableaux for verifying stream authentication protocols". In order to do this, they first construct a combined logic by adding a temporal dimension to the logic of beliefs by using the fibring method to combine logics, as introduced by Gabbay and colleagues. A sound and complete logic is provided, and, as the cherry on the cake, the system is applied to the authors' formalization of the TESLA authentication protocol, that also served as a successful jumping-off point for Wozna and Lomuscio's "A complete and decidable security-specialised logic and its application to the TESLA protocol", published in the proceedings of AAMAS'06.

Similarly, Teepe combines a protocol and a security-inspired logic in his "On BAN logic and hash functions or: How an unjustified inference rule causes problems". He gives a short introduction to cryptographic hash functions and then shows, both proof-theoretically and semantically, that the well-known BAN logic proves a very undesirable conclusion about a simple communication protocol. The result does not invalidate logical approaches to protocol analysis, but does provide a welcome warning not to include too strong rules in order to make the logic complete.

Acknowledgments We would like to thank all the people who helped to bring about FAMAS'06. First of all, we thank all speakers for ensuring a diverse and interesting workshop. Special thanks are due to the members of the program committee: Hans van Ditmarsch, Amal El-Fallah-Seghrouchni, Andreas Herzig, 
Wiebe van der Hoek, Wojtek Jamroga, John-Jules Meyer, Michael Luck, Chris Reed, David Robertson, Jeff Rosenschein, Carles Sierra, and Onn Shehory. We thank them and a number of anonymous referees for their professionalism and their dedication to select papers of quality and to provide authors with useful, constructive feedback during the in-depth reviewing process. The proceedings and website would not exist without the help of Marcin Dziubiński and Mateusz Srebrny in Warsaw.

Open Access This article is distributed under the terms of the Creative Commons Attribution Noncommercial License which permits any noncommercial use, distribution, and reproduction in any medium, provided the original author(s) and source are credited. 